\title{
Comportamentos em saúde de uma população portadora do HIV/Aids
}

\author{
Health Behavior in a population with HIVIAids \\ Comportamientos en salud de una población portadora de HIV/Aids
}

\author{
Tiago Cristiano Lima', Maria Isabel Pedreira Freitas" \\ 'Universidade Estadual de Campinas, Hospital das Clínicas, Unidade de Moléstias Infecciosas e Parasitárias. \\ Campinas-SP, Brasil. \\ "Universidade Estadual de Campinas, Faculdade de Ciências Médicas, Departamento de Enfermagem. \\ Campinas-SP, Brasil.
}

Submissão: 20-12-2010 Aprovação: 27-12-2011

\section{RESUMO}

Estudo descritivo exploratório, com abordagem quantitativa e corte transversal, cujo objetivo foi identificar comportamentos de idosos portadores do HIV/Aids e analisar associação com sexo. Utilizou-se instrumento testado e validado quanto ao conteúdo. As variáveis foram analisadas pelos testes Qui-quadrado e Exato de Fisher. Participaram cento e nove pessoas. Os resultados revelaram que eram sexualmente ativos $(57,8 \%)$, heterossexuais $(85,5 \%)$, com companheiro fixo $(66,1 \%)$. Relataram dificuldade para utilizar o preservativo $(22,2 \%$ dos homens) pelo comprometimento da ereção; $97,2 \%$ referiram não usar drogas para estimulação sexual; 90,8\% afirmaram não ter usado proteção nas relações antes de se saberem infectados. Trata-se de população sexualmente ativa, que passou a utilizar proteção nas relações sexuais após saber da infecção.

Descritores: HIV; Aids; Enfermagem; Comportamento.

\begin{abstract}
This is a descriptive exploratory research, with quantitative approach and transversal cut. The purpose was to identify behaviors in aged population HIV/Aids infected and also to analyze association with sex. Regarding the content, a tested and validated instrument was used. The variables were analyzed with qui-square and Fisher test. A hundred and nine individuals were interviewed. The results revealed them as sexually active $(57,8 \%)$, heterosexual $(85,5 \%)$, with fix partner $(66,1 \%)$. They reported difficulty in using condoms (22,2\% of men) due to erection issues; $97,2 \%$ referred not using drugs for sexual stimulation; $90,8 \%$ of them reported not having used protection in sexual relations before being aware of the infection. It was concluded that the population were sexually active, and that they started using protection, in their sexual relations, after awareness of the infection. Key words: HIV; Aids; Nursing; Behavior.
\end{abstract}

\section{RESUMEN}

Estudio descriptivo exploratorio, con abordaje cuantitativo y corte transversal, cuyo objetivo fue identificar comportamientos de una población de ancianos, portadora de HIV/Aids, y analizar asociación con género. Se utilizó un instrumento probado y convalidado en relación al contenido. Las variables fueran analizadas con testes de Qui-cuadrado y de Fisher. Ciento nueve pacientes fueron entrevistados. Los resultados revelaron una población sexualmente activa (57,8\%); heterosexuales $(85,5 \%)$; y con compañero fijo $(66,1 \%)$. Relataron dificultad para uso del preservativo $(22,2 \%$ de los hombres) por el compromiso con la erección; 97,2 \% relataron no usar drogas para estimulación sexual; 90,8\% afirman no haber usado protección en las relaciones antes de saber que estaban infectados. Concluimos que se trata de una población de personas sexualmente activas, que pasó a usar protección, en las relaciones sexuales, después de saber que estaban infectados.

Palabras clave: VIH, Aids, Enfermería, Conducta.

Trabalho extraído de Dissertação de Mestrado apresentada à Universidade Estadual de Campinas, Faculdade de Ciência Médicas, Programa de Pós Graduação em Enfermagem, Campinas-SP, Brasil, 2009.

\section{AUTOR CORRESPONDENTE Tiago Cristiano Lima E-mail: doslima@hotmail.com}




\section{INTRODUÇÃO}

A epidemia causada pelo Vírus da Imunodeficiência Humana (HIV) representa fenômeno global, dinâmico e instável, cuja forma de ocorrência nas diferentes regiões do mundo depende, dentre outros fatores, do comportamento humano individual e coletivo. A Síndrome da Imunodeficiência Adquirida (Aids) destaca-se entre as enfermidades infecciosas por sua magnitude e pela extensão dos danos causados às populações. Suas características e repercussões vêm sendo discutidas e estudadas pela comunidade científica e pela sociedade em geral ${ }^{(1)}$.

De acordo com relatório do Programa Conjunto das Nações Unidas sobre HIV/Aids (UNAIDS), estima-se que existam 35,7 milhões de pessoas com HIV/Aids em todo o mundo ${ }^{(2)}$.

A introdução da terapia antirretroviral de alta potência, somada às ações de prevenção e controle da infecção pelo vírus da Aids, tem resultado em alterações no padrão da epidemia $^{(3)}$. Observa-se uma mudança na história natural da doença, passando de um desfecho letal rápido com características uniformes para uma doença controlável, com caráter crônico, tendo atualmente evolução prolongada ${ }^{(4)}$.

A infecção pelo HIV/Aids, vista a princípio como específica de indivíduos jovens e considerados de grupos de risco passou a atingir qualquer pessoa da sociedade, independente de sexo e idade, sendo a via de transmissão heterossexual uma importante característica na dinâmica da epidemia ${ }^{(5-6)}$.

O crescimento no número de casos de HIV/Aids, em pessoas com 50 anos ou mais, representa a mais nova característica da epidemia ${ }^{(6-8)}$. Entre os anos de 1996 a 2006, na faixa etária de 50-59 anos, a taxa de incidência entre os homens passou de 17,9 para 29,3; entre as mulheres, cresceu de 6,0 para 17,3 . Houve, no mesmo período, um aumento na taxa de incidência entre indivíduos com mais de 60 anos. Nos homens o índice passou de 5,9 para 8,8 e nas mulheres, cresceu de 1,7 para $5,1^{(9)}$. Esse fato pode estar relacionado à nova geração de idosos que têm recursos para prolongar a qualidade de vida, o que consequentemente prolonga também a vida sexual, associado à existência do tabu de se falar sobre sexualidade nesta faixa etária. Esses casos de infecção pelo HIV ocorrem na maioria das vezes por contaminação sexual e, quase sempre, entre heterossexuais ${ }^{(7-8)}$.

São fatores de risco para transmissão e contaminação pelo vírus, nessa população: o aumento do número de pessoas idosas sexualmente ativas, porém com prática sexual não segura (não utilizam proteção nas relações sexuais) ${ }^{(10-11)}$; uso de bebida alcoólica e de drogas ${ }^{(12)}$; falta de conhecimento em relação aos riscos para contaminação pelo HIV/Aids e à necessidade de prevenção ${ }^{(13)}$; despreparo dos profissionais de saúde para identificar a pessoa idosa como sexualmente ativa, desperdiçando a oportunidade de oferecer as informações necessárias para prevenção da doença ${ }^{(13)}$; preconceito e estigma para com esta população, por parte dos familiares e amigos, no que se refere à sexualidade e à presença de doença sexualmente transmissível ${ }^{(10-11)}$.

Do ponto de vista da elaboração e execução de políticas públicas de saúde, torna-se fundamental conhecer a mudança no perfil da doença, assim como as características e comportamentos individuais e coletivos apresentados pelas pessoas infectadas. É também importante considerar que o planejamento das ações de assistência e de educação em saúde a estes pacientes compreende um processo dinâmico e contínuo, que objetiva capacitar indivíduos e/ou grupos para refletirem criticamente sobre os problemas de saúde e buscarem soluções para eles, considerando os aspectos sócio-econômico-cultural e político que estão à sua volta(4,14).

A mudança no perfil da epidemia torna essencial o levantamento de dados, para estimar o risco a que essa população está exposta. As intervenções devem ser planejadas considerando-se os comportamentos de saúde destes sujeitos, o que exige profissionais críticos, abertos a novos valores, capacitados sobre a complexidade do vírus e da doença por ele provocada, com conhecimento das políticas de saúde. Soma-se a isso a necessidade de desenvolvimento e implementação progressiva de estratégias abrangentes de cuidado e suporte a indivíduos, famílias e comunidades afetadas pelo HIV/Aids ${ }^{(15)}$.

\section{OBJETIVOS}

Identificar comportamentos de saúde de uma população portadora do HIV/Aids, com 50 anos ou mais, e analisar a associação destes comportamentos com o sexo.

\section{METODOLOGIA}

Estudo do tipo descritivo exploratório, com abordagem quantitativa e corte transversal. A população foi constituída por pacientes ambulatoriais do complexo de atendimento à saúde da Universidade Estadual de Campinas, no interior do Estado de São Paulo, Brasil.

Foram incluídos no estudo pacientes soropositivos para o HIV/Aids, com 50 anos ou mais, que assinaram o Termo de Consentimento Livre e Esclarecido, após terem sido orientados sobre a pesquisa, com capacidade cognitiva para entender com clareza as perguntas feitas pelo pesquisador e respondê-las. Foram excluídos do estudo os pacientes que se recusaram a assinar o Termo de Consentimento Livre e Esclarecido e os que haviam participado do teste piloto durante o processo de elaboração do instrumento para coleta de dados.

O tamanho da amostra foi determinado pelo número de pacientes atendidos no período de tempo estabelecido de quatro meses, compreendido entre outubro de 2008 e janeiro de 2009. O instrumento utilizado para a entrevista foi testado e validado por peritos quanto ao seu conteúdo em etapa anterior $^{(16)}$. Teve por objetivo caracterizar a população de idosos com 50 anos ou mais, portadora do HIV/Aids, sociodemográfica e clinicamente quanto aos comportamentos de saúde e quanto às crenças e atitudes relacionadas à doença e ao tratamento. Os pacientes foram abordados individualmente em local privativo e esclarecidos quanto ao objetivo do trabalho. A todos os participantes foi solicitada a assinatura do Termo de Consentimento Livre e Esclarecido, sendo assegurado o direito de recusar-se a participar da pesquisa ou retirar-se dela a qualquer tempo, sem que houvesse qualquer risco ou prejuízo. Após a anuência, foi realizada a entrevista. 
Os dados inseridos em planilha do programa Microsoft Excel foram posteriormente analisados, utilizando-se o programa computacional SPSS for Windows, versão 10.0 e 15.0 (SPSS Inc, 1989-1999, Chicago, IL, USA), sob orientação do Serviço de Estatística da Comissão de Pesquisa da Faculdade de Ciências Médicas, da Universidade Estadual de Campinas.

Utilizaram-se os testes estatísticos Qui-quadrado ou Exato de Fisher, quando indicado, para análise de associação e comparação entre a variável sexo e as variáveis referentes aos comportamentos de saúde. O nível de significância adotado foi de $5 \%$.

O projeto de pesquisa foi aprovado pelo Comitê de Ética em Pesquisa da Faculdade de Ciência Médicas da Universidade Estadual de Campinas, sob parecer no 275/2007, homologado em reunião realizada em 26 de junho de 2007.

\section{RESULTADOS}

A amostra foi composta por 109 sujeitos. Observou-se que, ao final do quarto mês, quase todos os pacientes que se apresentavam para serem atendidos nos ambulatórios já haviam participado da entrevista, não havendo mais novos casos. $\mathrm{O}$ período aproximado para aplicação do instrumento variou de 20 a 40 minutos.
O instrumento de coleta de dados foi composto pelas sessões: caracterização sociodemográfica, caracterização clínica, comportamentos de saúde e crenças e atitudes sobre a doença e o tratamento. Neste estudo, serão apresentados os dados referentes à sessão de comportamentos de saúde, por considerar-se este aspecto relevante para o direcionamento da educação em saúde oferecida a estes pacientes no que diz respeito à prevenção.

A população estudada foi composta predominantemente por sujeitos do sexo masculino (57,8\%); com idade média de 55,78 anos (DP: 5,10), variando entre 50 e 74 anos; a média de anos completos de estudo foi de 6,13 (DP: 3,67), com um mínimo de 1 ano e máximo de 12; a renda familiar média referida foi de $\mathrm{R} \$ 1486,93$ reais, com um valor mínimo de $\mathrm{R} \$$ 300 e um valor máximo de $\mathrm{R} \$ 8000$ reais; sem união estável $(65,1 \%)$; em sua maioria católica $(65,1 \%)$. 81,7\% referiram residir com uma ou mais pessoas sendo os responsáveis pela manutenção do domicílio.

A análise do comportamento de saúde da clientela entrevistada mostrou que poucos faziam uso de bebida alcoólica $(12,8 \%)$, cigarro $(3,7 \%)$ e nenhum referiu fazer uso de drogas. Relataram ser sexualmente ativos $(57,8 \%)$, heterossexuais $(85,5 \%)$, com companheiro fixo $(66,1 \%)$ e vivendo sobre o mesmo teto $(68,3 \%)$. Dos sexualmente ativos, $66,1 \%$ referiram

Tabela 1 - Comportamentos de saúde em função do sexo. Campinas - SP, 2008 - 2009

\begin{tabular}{|c|c|c|c|c|c|c|}
\hline \multirow{2}{*}{ VARIÁVEIS } & \multirow{2}{*}{ CATEGORIAS } & \multicolumn{2}{|c|}{ MASCULINO } & \multicolumn{2}{|c|}{ FEMININO } & \multirow{2}{*}{ P-valor } \\
\hline & & $N$ & $\%$ & $\mathrm{~N}$ & $\%$ & \\
\hline $\begin{array}{l}\text { Companheiro fixo } \\
\qquad(\mathrm{n}=63)\end{array}$ & $\begin{array}{l}\text { Não } \\
\text { Sim }\end{array}$ & $\begin{array}{l}20 \\
28\end{array}$ & $\begin{array}{l}41,7 \\
58,3\end{array}$ & $\begin{array}{l}01 \\
13\end{array}$ & $\begin{array}{l}07,1 \\
92,9\end{array}$ & $0,023 *$ \\
\hline $\begin{array}{l}\text { Relação sexual } \\
\text { atualmente } \\
(\mathrm{n}=109)\end{array}$ & $\begin{array}{l}\text { Não } \\
\text { Sim }\end{array}$ & $\begin{array}{l}15 \\
48\end{array}$ & $\begin{array}{l}23,8 \\
76,2\end{array}$ & $\begin{array}{l}31 \\
15\end{array}$ & $\begin{array}{l}67,4 \\
32,6\end{array}$ & $0,001^{+}$ \\
\hline $\begin{array}{l}\text { Relação sexual com } \\
\text { mais de uma pessoa } \\
\text { atualmente }(n=63)\end{array}$ & $\begin{array}{l}\text { Não } \\
\text { Sim }\end{array}$ & $\begin{array}{l}28 \\
20\end{array}$ & $\begin{array}{l}58,3 \\
41,7\end{array}$ & $\begin{array}{l}13 \\
01\end{array}$ & $\begin{array}{l}92,9 \\
07,1\end{array}$ & $0,023 *$ \\
\hline $\begin{array}{l}\text { Relação sexual com } \\
\text { mais de uma pessoa } \\
\text { antes de se saber } \\
\text { portador do HIV/Aids } \\
\qquad(\mathrm{n}=109)\end{array}$ & $\begin{array}{l}\text { Não } \\
\text { Sim }\end{array}$ & $\begin{array}{l}14 \\
49\end{array}$ & $\begin{array}{l}22,2 \\
77,8\end{array}$ & $\begin{array}{l}37 \\
09\end{array}$ & $\begin{array}{l}80,4 \\
19,6\end{array}$ & $0,001^{+}$ \\
\hline $\begin{array}{l}\text { Utilizava proteção nas } \\
\text { relações sexuais antes } \\
\text { de se saber portador } \\
\text { do HIV/Aids }(\mathrm{n}=109)\end{array}$ & $\begin{array}{l}\text { Não } \\
\text { Sim }\end{array}$ & $\begin{array}{l}53 \\
10\end{array}$ & $\begin{array}{l}84,1 \\
15,9\end{array}$ & $\begin{array}{l}46 \\
00\end{array}$ & $\begin{array}{c}100 \\
00\end{array}$ & $0,005 *$ \\
\hline $\begin{array}{l}\text { Razão de não } \\
\text { utilizar proteção no } \\
\text { relacionamento sexual } \\
(\mathrm{n}=100)\end{array}$ & $\begin{array}{l}\text { Confiava no/a parceiro/a } \\
\text { Falta de conhecimento sobre a doença e a necessidade de proteção } \\
\text { Devido ao uso excessivo de bebida alcoólica } \\
\text { Por descuido próprio } \\
\text { Devido a falta do preservativo na hora da relação } \\
\text { Parceiro/a não aceitava e/ou não gostava do preservativo }\end{array}$ & $\begin{array}{l}09 \\
16 \\
03 \\
12 \\
13 \\
01\end{array}$ & $\begin{array}{l}16,7 \\
29,6 \\
05,6 \\
22,2 \\
24,1 \\
01,9\end{array}$ & $\begin{array}{l}24 \\
09 \\
01 \\
05 \\
00 \\
07\end{array}$ & $\begin{array}{l}52,2 \\
19,6 \\
02,2 \\
10,9 \\
00 \\
15,2\end{array}$ & $0,001 *$ \\
\hline
\end{tabular}

* Teste Exato de Fisher ${ }^{+}$Teste do Qui - quadrado 
não estar se relacionando com mais de uma pessoa no período da entrevista e $83,9 \%$ faziam uso de proteção nas relações. Destes, 22,2\% disseram ter dificuldade para utilizar o preservativo, principalmente, devido ao comprometimento da ereção. $97,2 \%$ referiram não usar drogas para estimulação sexual.

Quando questionados sobre a vida sexual pregressa, $53,2 \%$ disseram ter se relacionado com mais de uma pessoa antes de se saberem portadores do vírus e 90,8\% afirmaram não ter feito uso de proteção. As principais razões citadas para o fato foram a confiança no parceiro $(30,3 \%)$ e a falta de conhecimento sobre a doença associado à necessidade de usar proteção $(22,9 \%)$.

Observou-se nessa população que o número de homens sexualmente ativos é maior do que o de mulheres $(p=$ 0,001), porém o número de mulheres com relacionamento fixo é maior do que o de homens ( $p=0,023$ ) (Tabela 1).

Uma característica identificada no estudo, tanto no momento da entrevista quanto antes dos sujeitos terem o conhecimento do diagnóstico de HIV/Aids, foi o fato de os homens $(47,1 \%$ atualmente e $77,8 \%$ antes do diagnóstico) se relacionarem com mais de uma pessoa mais frequentemente do que as mulheres $(07,1 \%$ atualmente e $19,6 \%$ antes do diagnóstico) ( $p=0,023$ atualmente e $p=0,001$ antes do diagnóstico). Em relação ao uso de proteção nas relações sexuais, antes de se saberem portadores do HIV/Aids, poucos eram os homens $(15,9 \%)$ e as mulheres (00\%) que utilizavam. No entanto, a frequência de mulheres que se protegiam durante a relação sexual era ainda menor ( $p=0,005)$, visto que nenhuma relatou o uso de proteção nas relações sexuais antes de se saber portadora de HIV/Aids. A justificativa para essa atitude difere entre os sexos. Os homens relataram não utilizar proteção principalmente pela falta do preservativo no momento da relação e por desconhecerem a doença e seus riscos, enquanto que as mulheres não a utilizavam por confiar nos seus parceiros.

Embora o problema de ereção associado ao uso do preservativo tenha sido mencionado como uma dificuldade para sua utilização, ele não foi mencionado no momento da entrevista como razão para a não utilização da proteção.

\section{DISCUSSÃO}

Ao se iniciar esse estudo, a maior preocupação foi a identificação dos principais comportamentos de saúde apresentados por esta população, com 50 anos ou mais portadora de HIV/Aids, a fim de direcionar o planejamento para a assistência à saúde, voltado às particularidades apreendidas. No entanto, no decorrer do trabalho, observou-se que não somente os indivíduos sabidamente contaminados necessitam atenção individualizada, como também, e talvez principalmente, os idosos não contaminados. Há necessidade de se ter esse olhar diferenciado do modelo biológico hegemônico, para estabelecer uma assistência voltada às suas reais características.

O contato do pesquisador com os sujeitos do estudo durante a aplicação do questionário concretizou a importância desse novo olhar na medida em que se processava a coleta de dados. A forma fluida, sincera e aberta com que as pessoas participaram surpreendeu os idealizadores do estudo. Em ambiente público, com o pequeno resguardo possível da individualidade, os entrevistados expunham temores, vivências, crenças e atitudes que extrapolavam as perguntas do questionário. Após finalizar a entrevista permaneciam em diálogo.

Um importante problema encontrado, quando se pensa na redução de riscos para contaminação pelo HIV em pessoas acima de 50 anos, está na inadequação da linguagem utilizada e no preconceito de acreditar que a Aids ainda é restrita aos mais jovens ${ }^{(12-13)}$. Com receio de que o paciente se sinta constrangido em falar sobre sua sexualidade, é desconsiderada, pelo profissional de saúde, a possibilidade de uma pessoa idosa ter HIV/Aids e por isso perde-se a oportunidade de orientá-la e até solicitar o teste diagnóstico para detecção precoce da doença ${ }^{(17)}$. Um estudo constatou que o profissional de saúde não acredita que o idoso possa estar infectado com o HIV e por isso não pede o exame imediatamente diante dos primeiros sintomas ${ }^{(18)}$.

O profissional de enfermagem, com freqüência, reluta em abordar a história e o estilo de vida sexual dos pacientes ou o faz de forma inadequada ${ }^{(19)}$. Em estudo com mulheres portadoras de problemas cardíacos, as autoras observaram que $76 \%$ das 170 pacientes estudadas preferiam que as discussões sobre atividade sexual e sexualidade fossem iniciadas por profissionais de saúde, no entanto, apenas 3\% haviam tido essa oportunidade ${ }^{(19)}$. Assim, quando não se toma a história sexual de um paciente, a abordagem está sendo incompleta e pode carecer de informações vitais que podem afetar a sua recuperação.

Os profissionais de saúde têm dificuldade em reconhecer que a sexualidade na pessoa idosa é uma realidade, o que os impede de incorporar a necessidade de medidas de prevenção voltadas a esta população(20). Acredita-se que a sexualidade não seja investigada, pois existe na sociedade a noção de que o sexo, a sexualidade, não existem na velhice ${ }^{(8)}$. No presente estudo, observou-se que $57,8 \%$ dos entrevistados referiram ser sexualmente ativos.

No início da epidemia de HIV/Aids, no Estado de São Paulo, a imensa maioria dos casos ocorria em homens e os coeficientes de incidência no sexo masculino eram expressivamente maiores do que no sexo feminino. Ocorre que houve um aumento tão importante do número de casos entre mulheres que a relação masculino/feminino, que em 1984 era de 27/1, aproximou-se de 2/1 em 1997 e vem se mantendo neste patamar apresentando, a partir de então, ligeira queda ano a ano ${ }^{(5)}$. Nesse estudo, observou-se um pequeno predomínio de pessoas do sexo masculino (57,8\%). Verificou-se, também, o predomínio $(65,1 \%)$ de pessoas sem união estável (solteiros, separados e viúvos); residentes com uma ou mais pessoas $(81,7 \%)$; e responsáveis pelo sustento da casa $(69,7 \%)$. Em estudo realizado com idosos portadores de HIV/Aids no Rio de Janeiro, o autor verificou que $71 \%$ dos sujeitos não tinham união estável por serem solteiros, viúvos ou separados ${ }^{(8)}$.

Observou-se maior proporção de homens que se relacionavam com mais de uma pessoa $(41,7 \%)$; e maior proporção de mulheres que não faziam uso de proteção nas relações sexuais antes de se saberem portadoras do HIV/Aids (100\%). 
Em estudo cujo objetivo foi analisar o comportamento, conhecimento e percepção de risco às DSTs/Aids em pessoas com 50 anos ou mais de idade, verificou-se que, com relação à prática de sexo seguro, os entrevistados demonstraram conhecer a importância de usar o preservativo para prevenção contra as DSTs/Aids ${ }^{(20)}$. No entanto, 78,5\% dos homens e $86,5 \%$ das muIheres não haviam utilizado o preservativo nas últimas relações sexuais. Tais atitudes podem ser atribuídas a fatores como: a prática não faz parte da cultura dos idosos; as mulheres ainda consideram o uso do preservativo como um método com objetivo de prevenir a gravidez; as mulheres confiam no parceiro ${ }^{(20-21)}$.

Estudo epidemiológico para avaliar o conhecimento sobre HIV/Aids de participantes de grupos de convivência do Vale dos Sinos, RS, Brasil, demonstrou que a maior parte das pessoas sabia que o uso do preservativo impedia a transmissão do HIV, porém, mais de $80 \%$ não o utilizavam. Além disso, os pesquisados não citaram a Aids como um problema de saúde pública e não manifestaram interesse na mudança de comportamento para sua prevenção ${ }^{(22)}$.

Cinco fatores de risco individuais para infecção pelo HIV/ Aids foram identificados em um estudo com idosos portadores da doença: abuso de álcool e drogas, desconhecimento da história de risco para infecção pelo HIV/Aids de seus parceiros, alterações psicológicas (oriundas de violência doméstica, crises familiares e abusos sexuais), não se proteger nas relações sexuais em consideração ao parceiro e falta de informação para prevenção contra o HIV/Aids ${ }^{(11)}$. Outros fatores, apontados como de maior vulnerabilidade entre as mulheres frente à doença, foram: escolaridade mais baixa, entendida como condição sócio-econômica menos privilegiada e parceiros com múltiplas parceiras sexuais ${ }^{(5)}$.

No presente estudo, dez pacientes referiram não ter estudado e os que o fizeram completaram em média 6,13 anos de estudo. Em outro estudo, a maioria dos indivíduos teve baixa escolaridade, tendo cursado no máximo, ensino fundamental ou primeiro grau ${ }^{(23)}$.

O nível educacional proporciona diferenças de acesso à informação e possibilidade de se beneficiar de novos conhecimentos $^{(23)}$. É inegável que alguns parâmetros para alcançar maior qualidade de prevenção e assistência, tais como o acesso à educação e aos métodos preventivos, estão diretamente ligados à situação sócio-econômica da população.

Assim, ao planejar a assistência ao idoso, seja ele portador ou não de HIV/Aids, estas questões devem ser levadas em consideração. Corrobora esta discussão um estudo em que foram avaliados trabalhos que abordavam a educação das pessoas mais velhas para reduzir os fatores de risco para contaminação pelo HIV/Aids ${ }^{(12-13)}$. Os autores verificaram a necessidade de discutir e adequar as formas utilizadas para abordar pessoas mais velhas sobre o assunto, uma vez que esta população apresenta alterações em seu estado geral de vida que necessitam ser consideradas, alterações essas fisiológicas e advindas de questões culturais, sociais, dentre outras.

Assistir uma pessoa que entrou no processo de envelhecimento exige preparo e competência do profissional de saúde, uma vez que a pessoa vive e teme formas sociais de discriminação. Se o idoso é portador de HIV/Aids, a situação se agrava, na medida em que traz consigo as representações sociais sobre a doença e, não raro, se depara com atitudes discriminatórias fundadas nelas. Sua situação de vida torna-se pautada pelo isolamento social, medo de ser discriminado, falta de trabalho e de perspectivas concretas no cotidiano, além das dificuldades para manter o tratamento. Há, também, desconfiança em relação aos profissionais de saúde que o atendem ${ }^{(18)}$.

As informações para a prevenção do HIV/Aids em idosos terão de levar em conta, além da desconstrução de imagens estereotipadas da doença no início da epidemia, fatores específicos dessa idade, como a dificuldade de mudança de hábitos e incorporação de novas formas de lidar com a sexualidade, além de outros importantes, como valores culturais, por exemplo ${ }^{(8)}$.

Assim, a assistência à nova geração de idosos, sexualmente ativos, cercados por tabus, preconceitos e crenças errôneas exige a atitude de profissionais cada vez mais capacitados, abertos a novos paradigmas e com conhecimento das particularidades apresentadas por eles. Desta forma, estarão preparados para oferecer uma abordagem segura e responsável aos idosos, principalmente no que tange à sexualidade e, consequentemente, agir prevenindo doenças evitáveis, o que parece ser um grande desafio para os profissionais de saúde.

\section{CONCLUSÃO}

A população estudada foi composta predominantemente por homens, sexualmente ativos, que fazia pouco uso de cigarro e bebidas alcoólicas e que passaram a utilizar proteção nas relações sexuais após se saberem infectados pelo HIV/Aids. Referiram não ter feito uso de proteção nas relações sexuais na vida pregressa por não estarem portando preservativos no momento da relação. Para as mulheres, a justificativa para o não uso do preservativo, foi o fato de confiarem nos parceiros. Cabe ressaltar que a grande maioria tinha companheiro fixo.

A sexualidade está presente nos idosos. Cabe aos profissionais de saúde reconhecer tal fato para agirem de forma a prevenir doenças sexualmente transmissíveis, visto que, estes ainda estão muito condicionados a lidar com doenças consideradas próprias da velhice.

\section{REFERÊNCIAS}

1. Brito AM, Castilho EA, Szwarcwald CL. AIDS e infecção pelo HIV no Brasil: uma epidemia multifacetada. Rev Soc Bras Med Trop 2001;34(2):207-17.

2. Programa Conjunto das Nações Unidas sobre HIV/aids
- UNAIDS (Brasil). Boletim epidemiológico. Brasília (DF); 2007.

3. Dourado I, Veras MASM, Barreira D, Brito AM. Tendências da epidemia de Aids no Brasil após a terapia 
anti-retroviral. Rev Saúde Pública 2006;40(1):9-17.

4. Pereira LCA, Machado LJ, Rodrigues RN. Perfil de causas múltiplas de morte relacionadas ao HIV/AIDS nos municípios de São Paulo e Santos, Brasil, 2001. Cad Saúde Pública 2007;23(3):645 -55.

5. Santos NJS, Tavra A, Silva SR, Buchalla CM, Laurenti R. A Aids no estado de São Paulo: as mudanças no perfil da epidemia e perspectivas da vigilância epidemiológica. Rev Bras Epidemiol 2002;5(2):12-7.

6. Araújo VLB, Brito DMS, Gimeniz MT, Queiroz TA, Tavares CM. Características da Aids na terceira idade em um hospital de referência do estado do Ceará, Brasil. Rev Bras Epidemiol 2007;10(4):544-54.

7. Gross JB. Estudo de pacientes portadores de HIV/Aids após os 60 anos de idade em duas unidades de saúde do estado do Rio de Janeiro. Rio de Janeiro. Dissertação [Mestrado em Medicina] - Fundação Oswaldo Cruz; 2005.

8. Zornitta M. Os novos idosos com Aids: sexualidade e desigualdade à luz da bioética. Rio de Janeiro. Dissertação [Mestrado em Ciências da Saúde] - Fundação Oswaldo Cruz; 2008.

9. Ministério da Saúde (Brasil). Boletim epidemiológico AIDS. Coordenação Nacional de Doenças Sexualmente Transmissíveis e AIDS. Ministério da Saúde: Brasília; 2008.

10. Silva CM, Vargens OMC. A percepção de mulheres quanto à vulnerabilidade feminina para contrair DST/HIV. Rev Esc Enferm USP 2009;43(2):401-6.

11. Neundorfer MM, Harris PB, Britton PJ, Lynch DA. HIVrisk factors for midlife and older womwn. Gerontologist 2005;45(5):617-25.

12. Savasta AM. HIV: associated transmission risks in older adults - an integrative review of the literature. J Assoc Nurses AIDS Care 2004;15(1):50-9.

13. Orel NA, Apencer M, Steele J. Getting the Message out to older adults: effective HIV health education risk reduction publications. J Appl Gerontol 2005; 24(5):490-508.

14. Madureira VSF, Trentini M. Relações de poder na vida conjugal e prevenção da AIDS. Rev Bras Enferm 2008;61(5):637-42.

15. Gabriel R, Barbosa DA, Vianna LAC. Perfil epidemiológico dos clientes com HIV/AIDS da unidade ambulatorial de hospital escola de grande porte - município São Paulo. Rev Latinoam Enferm 2005;13(4):509-13.

16. Lima TC, Freitas MIP. Elaboração, validação e aplicação de um instrumento para caracterização de uma população com 50 anos ou mais portadora do HIV/Aids. Campinas. Dissertação [Mestrado em Enfermagem] Universidade Estadual de Campinas; 2009.

17. Inelmen EM, Gasparine G, Enzi G. HIV/Aids in older adults. Geriatrics 2005;60(9):26-30.

18. Brasileiro M, Freitas MIF. Social representations about Aids in people over 50, infected by HIV. Rev Latinoam Enferm 2006;14(5):789-95.

19. Warner $\mathrm{PH}$, Rowe $\mathrm{T}$, Whipple $\mathrm{B}$. Thedding light on the sexual history. Am J Nurs 1999;99(6):34-40.

20. Olivi M, Santana RG, Mathias TAF. Behavior, knowledge and perception of risks about sexually transmitted diseases in a group of people over 50 years old. Rev Latinoam Enferm 2008;16(4):679-85.

21. Cechim PL, Selli L. Mulheres com HIV/AIDS: fragmentos de sua face oculta. Rev Bras Enferm 2007;60(2):145-9.

22. Lazzarotto AR, Kramer AS, Hadrich M, Tonin M, Caputo $\mathrm{P}$, Sprinz E. O conhecimento de HIV/Aids na terceira idade: estudo epidemiológico no Vale dos Sinos, Rio Grande do Sul, Brasil. Cien Saúde Coletiva 2008; 13(6):1833-40.

23. Pottes FA, Brito AM, Gouveia GC, Araújo AC, Carneiro RM. Aids e envelhecimento: características dos casos com idade igual ou maior que 50 anos em Pernambuco, de 1990 a 2000. Rev Bras Epidemiol 2007; 10(3):338-51. 